PROCEEDINGS OF THE

AMERICAN MATHEMATICAL SOCIETY

Volume 135, Number 8, August 2007, Pages 2599-2606

S 0002-9939(07)08778-3

Article electronically published on March 29, 2007

\title{
A NOTE ON SUBGAUSSIAN ESTIMATES FOR LINEAR FUNCTIONALS ON CONVEX BODIES
}

\author{
A. GIANNOPOULOS, A. PAJOR, AND G. PAOURIS
}

(Communicated by N. Tomczak-Jaegermann)

\begin{abstract}
We give an alternative proof of a recent result of Klartag on the existence of almost subgaussian linear functionals on convex bodies. If $K$ is a convex body in $\mathbb{R}^{n}$ with volume one and center of mass at the origin, there exists $x \neq 0$ such that

$$
\left|\left\{y \in K:|\langle y, x\rangle| \geq t\|\langle\cdot, x\rangle\|_{1}\right\}\right| \leq \exp \left(-c t^{2} / \log ^{2}(t+1)\right)
$$

for all $t \geq 1$, where $c>0$ is an absolute constant. The proof is based on the study of the $L_{q}$-centroid bodies of $K$. Analogous results hold true for general log-concave measures.
\end{abstract}

\section{INTRODUCTION}

The purpose of this note is to provide an alternative proof of a recent result of Klartag (see [9]) on the existence of almost subgaussian linear functionals on convex bodies. Let $K$ be a convex body in $\mathbb{R}^{n}$ with volume $|K|=1$ and center of mass at the origin. Let $\psi:[0, \infty) \rightarrow[0, \infty)$ be a convex, increasing function with $\psi(0)=0$. For every bounded measurable function $f: K \rightarrow \mathbb{R}$, define

$$
\|f\|_{\psi}=\inf \left\{t>0: \int_{K} \psi(|f(x)| / t) d x \leq 1\right\} .
$$

We will be interested in the $\psi_{\alpha}$-norm of linear functionals $y \mapsto\langle y, x\rangle$ on $K$, where $1 \leq \alpha \leq 2$ and $\psi_{\alpha}(t)=e^{t^{\alpha}}-1$. We say that $x \neq 0$ defines a $\psi_{\alpha}$-direction for $K$ with constant $B>0$ if

$$
\|\langle\cdot, x\rangle\|_{\psi_{\alpha}} \leq B\|\langle\cdot, x\rangle\|_{1} .
$$

It is not hard to check that this holds true if and only if

$$
\|\langle\cdot, x\rangle\|_{q} \leq c B q^{1 / \alpha}\|\langle\cdot, x\rangle\|_{1}
$$

for every $q \geq 1$, where $c>0$ is an absolute constant. By Borell's lemma (see [13, Appendix III), there exists an absolute constant $C>0$ such that if $K$ is a convex body in $\mathbb{R}^{n}$, then every $x \neq 0$ is a $\psi_{1}$-direction for $K$ with constant $C$.

Received by the editors April 20, 2006.

2000 Mathematics Subject Classification. Primary 52A20; Secondary 46 B07.

Key words and phrases. Isotropic convex bodies, concentration of volume, tail estimates for linear functionals, $L_{q}$-centroid bodies.

The project was co-funded by the European Social Fund and National Resources - (EPEAEK II) "Pythagoras II". The second named author would like to thank the Department of Mathematics of the University of Athens for the hospitality. The third named author was supported by a Marie Curie Intra-European Fellowship (EIF), Contract MEIF-CT-2005-025017. 
The study of $\psi_{2}$-directions for linear functionals on convex bodies is motivated by the study of isotropic convex bodies and Bourgain's approach to the isotropic constant problem. A convex body $K$ in $\mathbb{R}^{n}$ is called isotropic if it has volume $|K|=1$, center of mass at the origin, and there exists a constant $L_{K}>0$ such that

$$
\int_{K}\langle y, \theta\rangle^{2} d y=L_{K}^{2}
$$

for every $\theta \in S^{n-1}$. Every convex body with center of mass at the origin has a linear image which is isotropic (see [12]). This image is unique up to orthogonal transformations, and hence, the isotropic constant $L_{K}$ is well-defined for the linear class of $K$. The isotropic constant problem asks if there exists an absolute constant $C>0$ such that $L_{K} \leq C$ for every isotropic convex body in any dimension. One can easily see that $L_{K}=O(\sqrt{n})$ for every $K$. Uniform boundedness of $L_{K}$ is known for some classes of bodies: unit balls of spaces with 1-unconditional basis, zonoids and their polars, etc. Bourgain (see [4]) proved that $L_{K}=O(\sqrt[4]{n} \log n)$ and, very recently, Klartag (see [8]) improved this bound to $L_{K}=O(\sqrt[4]{n})$. Moreover, in [5] Bourgain proved that if every $x \neq 0$ is a $\psi_{2}$-direction for $K$ with constant $B$, then $L_{K}$ is bounded by $c B \log (B+1)$.

A question of Milman, related to this line of thought, is whether, for every isotropic convex body $K$ in $\mathbb{R}^{n}$, most $\theta \in S^{n-1}$ define a $\psi_{2}$-direction for $K$ with a "good" constant (for example, logarithmic in $n$ ). Until recently, it was not known if there exists an absolute constant $C>0$ such that every isotropic convex body has at least one $\psi_{2}$-direction with constant $C$. Some positive results are known for special classes of convex bodies. Bobkov and Nazarov (see [2] and [3]) have proved that if $K$ is an isotropic 1-unconditional convex body, then $\|\langle\cdot, x\rangle\|_{\psi_{2}} \leq c \sqrt{n}\|x\|_{\infty}$ for every $x \neq 0$. This shows that the diagonal direction is a $\psi_{2}$-direction. For the class of zonoids, the existence of good $\psi_{2}$-directions was established in [14]. Another partial result, which gives more information in the case of isotropic convex bodies with "small diameter", was obtained in [15]: If $K \subseteq\left(\gamma \sqrt{n} L_{K}\right) B_{2}^{n}$ for some $\gamma>0$, then

$$
\sigma\left(\theta \in S^{n-1}:\|\langle\cdot, \theta\rangle\|_{\psi_{2}} \geq c_{1} \gamma t L_{K}\right) \leq \exp \left(-c_{2} \sqrt{n} t^{2} / \gamma\right)
$$

for every $t \geq 1$, where $\sigma$ is the rotationally invariant probability measure on $S^{n-1}$ and $c_{1}, c_{2}>0$ are absolute constants.

Klartag (see [9]) gave a positive answer to this question, showing that every isotropic convex body admits at least one almost subgaussian linear functional. Our aim is to give a second (short) proof of this fact.

Theorem 1.1. Let $K$ be an isotropic convex body in $\mathbb{R}^{n}$. There exists $x \neq 0$ such that

$$
\left|\left\{y \in K:|\langle y, x\rangle| \geq t\|\langle\cdot, x\rangle\|_{1}\right\}\right| \leq \exp \left(-c t^{2} / \log ^{\tau}(t+1)\right)
$$

for all $t \geq 1$, where $c, \tau>0$ are absolute constants.

It is clear that if $x$ defines a $\psi_{\alpha}$-direction for $K$ and if $T \in S L(n)$, then $T^{*} x$ defines a $\psi_{\alpha}$-direction (with the same constant) for $T(K)$. It follows that Theorem 1.1 provides almost subgaussian directions for every convex body: If $K$ is a convex body in $\mathbb{R}^{n}$ with volume one and center of mass at the origin, there exists $x \neq 0$ such that (1.6) holds true for all $t \geq 1$.

The argument of Klartag is based on the study of the level sets of the logarithmic Laplace transform of log-concave functions. The argument we present here is based 
on the study of the $L_{q}$-centroid bodies of an isotropic convex body. This family of bodies was studied and used by the third named author in [15], and in particular in [16], where the following sharp dimension-dependent concentration of volume estimate was proved: There exists an absolute constant $c>0$ such that if $K$ is an isotropic convex body in $\mathbb{R}^{n}$, then

$$
\left|\left\{x \in K:\|x\|_{2} \geq c \sqrt{n} L_{K} t\right\}\right| \leq \exp (-\sqrt{n} t)
$$

for every $t \geq 1$, where $\|\cdot\|_{2}$ is the Euclidean norm. The tools which are developed in 16] allow us to give a very simple proof of Theorem 1.1. We present an argument which gives $\tau=2$, i.e. the upper bound in (1.6) is $\exp \left(-c t^{2} / \log ^{2}(t+1)\right)$.

Notation. We work in $\mathbb{R}^{n}$, which is equipped with a Euclidean structure $\langle\cdot, \cdot\rangle$. We denote by $\|\cdot\|_{2}$ the corresponding Euclidean norm, and write $B_{2}^{n}$ for the Euclidean unit ball, and $S^{n-1}$ for the unit sphere. Volume is denoted by $|\cdot|$. If $K$ is a convex body in $\mathbb{R}^{n}$, we set $\bar{K}=K /|K|^{1 / n}$; this is the dilation of $K$ which has volume one. We write $\sigma$ for the rotationally invariant probability measure on $S^{n-1}$. The Grassmann manifold $G_{n, k}$ of $k$-dimensional subspaces of $\mathbb{R}^{n}$ is equipped with the Haar probability measure $\mu_{n, k}$.

A convex body is a compact convex subset $C$ of $\mathbb{R}^{n}$ with non-empty interior. We say that $C$ has center of mass at the origin if $\int_{C}\langle x, \theta\rangle d x=0$ for every $\theta \in S^{n-1}$. The support function $h_{C}: \mathbb{R}^{n} \rightarrow \mathbb{R}$ of $C$ is defined by $h_{C}(x)=\max \{\langle x, y\rangle: y \in C\}$. The mean width of $C$ is defined by

$$
w(C)=\int_{S^{n-1}} h_{C}(\theta) \sigma(d \theta)
$$

The letters $c, c^{\prime}, c_{1}, c_{2}$, etc. denote absolute positive constants which may change from line to line. We refer to the books [18, [13] and [17] for basic facts from the Brunn-Minkowski theory and the asymptotic theory of finite dimensional normed spaces.

\section{Normalized $L_{q}$-CENTROID BOdies}

Let $K$ be a convex body of volume 1 in $\mathbb{R}^{n}$. For every $q \geq 1$ we define the $L_{q}$-centroid body $Z_{q}(K)$ of $K$ by its support function:

$$
h_{Z_{q}(K)}(x)=\|\langle\cdot, x\rangle\|_{q}:=\left(\int_{K}|\langle y, x\rangle|^{q} d y\right)^{1 / q} .
$$

Since $|K|=1$, we readily see that $Z_{1}(K) \subseteq Z_{p}(K) \subseteq Z_{q}(K) \subseteq Z_{\infty}(K)$ for every $1 \leq p \leq q \leq \infty$, where $Z_{\infty}(K)=\operatorname{conv}\{K,-K\}$. On the other hand, one has the reverse inclusions

$$
Z_{q}(K) \subseteq \frac{c q}{p} Z_{p}(K)
$$

for every $1 \leq p<q<\infty$, as a consequence of the $\psi_{1}$-behavior of $y \mapsto\langle y, x\rangle$. Observe that $Z_{q}(K)$ is always symmetric, and $Z_{q}(T K)=T\left(Z_{q}(K)\right)$ for every $T \in$ $S L(n)$ and $q \in[1, \infty]$. Also, if $K$ has its center of mass at the origin, then $Z_{q}(K) \supseteq$ $c Z_{\infty}(K)$ for all $q \geq n$, where $c>0$ is an absolute constant.

It should be mentioned that $L_{q}$-centroid bodies were introduced in [10] under a different normalization. Lutwak, Yang and Zhang (see [11] and [7] for a different proof) have established the $L_{q}$ affine isoperimetric inequality

$$
\left|Z_{q}(K)\right|^{1 / n} \geq\left|Z_{q}\left(\overline{B_{2}^{n}}\right)\right|^{1 / n} \geq c \sqrt{q / n}
$$


for every $1 \leq q \leq n$, where $c>0$ is an absolute constant.

We will need upper estimates for the quermassintegrals of the $L_{q}$-centroid bodies of an isotropic convex body. These follow immediately from estimates on the projections of $Z_{q}(K)$, which are obtained in [16]. Fix $1 \leq k \leq n$ and a $k$-dimensional subspace $F$ of $\mathbb{R}^{n}$, and denote by $E$ the orthogonal subspace of $F$. For every $\phi \in S_{F}$, define $E(\phi)=\{y \in \operatorname{span}\{E, \phi\}:\langle y, \phi\rangle \geq 0\}$. By a theorem of K. Ball (see [1] and 12]), for every convex body $K$ of volume one in $\mathbb{R}^{n}$, for every $q \geq 0$ and every $\phi \in F$, the function

$$
\phi \mapsto\|\phi\|_{2}^{1+\frac{q}{q+1}}\left(\int_{K \cap E(\phi)}|\langle y, \phi\rangle|^{q} d y\right)^{-\frac{1}{q+1}}
$$

is a gauge function on $F$ (see also [6] for the not necessarily symmetric case). If we denote by $B_{q}(K, F)$ the convex body in $F$ whose gauge function is defined by (2.4), then the volume of $B_{q}(K, F)$ is given by

$$
\left|B_{q}(K, F)\right|=\left|B_{2}^{k}\right| \int_{S_{F}}\left(\int_{K \cap E(\phi)}|\langle x, \phi\rangle|^{q} d x\right)^{\frac{k}{q+1}} d \sigma_{F}(\phi) .
$$

The following identity was proved in [16].

Proposition 2.1. Let $K$ be a convex body of volume 1 in $\mathbb{R}^{n}$ and let $1 \leq k \leq n-1$. For every $F \in G_{n, k}$ and every $q \geq 1$ we have that

$$
P_{F}\left(Z_{q}(K)\right)=(k+q)^{1 / q}\left|B_{k+q-1}(K, F)\right|^{1 / k+1 / q} Z_{q}\left(\bar{B}_{k+q-1}(K, F)\right) .
$$

Using this identity and exploiting (2.5) in order to estimate the volume of $B_{q}(K, F)$, one gets the following estimate (see [16]).

Proposition 2.2. Let $K$ be an isotropic convex body in $\mathbb{R}^{n}$. If $F \in G_{n, k}$ and $E=F^{\perp}$ then, for every $q \in \mathbb{N}$ we have that

$$
P_{F}\left(Z_{q}(K)\right) \subseteq \frac{c(k+q)}{k} L_{K} Z_{q}\left(\bar{B}_{k+q-1}(K, F)\right)
$$

where $c>0$ is an absolute constant.

Definition 2.3. Let $K$ be an isotropic convex body in $\mathbb{R}^{n}$. For every integer $q \geq 1$ we define the normalized $L_{q}$-centroid body $K_{q}$ of $K$ by

$$
K_{q}=\frac{1}{\sqrt{q} L_{K}} Z_{q}(K) \text {. }
$$

Since $\left|Z_{q}\left(\bar{B}_{k+q-1}(K, F)\right)\right| \leq\left|Z_{\infty}\left(\bar{B}_{k+q-1}(K, F)\right)\right| \leq 4^{k}$, Proposition 2.2 shows that

$$
\left|P_{F}\left(K_{q}\right)\right|^{1 / k} \leq \frac{c(k+q)}{k \sqrt{q}}\left|Z_{q}\left(\bar{B}_{k+q-1}(K, F)\right)\right|^{1 / k} \leq \frac{c_{1}(k+q)}{k} \frac{\sqrt{k}}{\sqrt{q}}\left|B_{2}^{k}\right|^{1 / k}
$$

for every $F \in G_{n, k}$. If $1 \leq k \leq q$, this estimate takes the simpler form

$$
\left|P_{F}\left(K_{q}\right)\right|^{1 / k} \leq 2 c_{1} \frac{\sqrt{q}}{\sqrt{k}}\left|B_{2}^{k}\right|^{1 / k}
$$

In particular, for every $F \in G_{n, q}$ we have

$$
\left|P_{F}\left(K_{q}\right)\right|^{1 / q} \leq 2 c_{1}\left|B_{2}^{q}\right|^{1 / q} .
$$


A standard argument implies that a similar estimate is valid for every $F \in G_{n, k}$, where $q \leq k \leq n$. To see this, observe that by the log-concavity of the quermassintegrals of $P_{F}\left(K_{q}\right)$ and by Kubota's formula,

$$
\left(\frac{\left|P_{F}\left(K_{q}\right)\right|}{\left|B_{2}^{k}\right|}\right)^{1 / k} \leq\left(\frac{\int\left|P_{H}\left(P_{F}\left(K_{q}\right)\right)\right| d \mu_{k, q}(H)}{\left|B_{2}^{q}\right|}\right)^{1 / q},
$$

where the integration is over all $q$-dimensional subspaces $H$ of $F$, and apply (2.11) pointwise taking into account the fact that $P_{H}\left(P_{F}\left(K_{q}\right)\right)=P_{H}\left(K_{q}\right)$. We summarize these observations in the next theorem.

Theorem 2.4. Let $K$ be an isotropic convex body in $\mathbb{R}^{n}$. If $1 \leq k, q \leq n$ are integers, and if $F \in G_{n, k}$, then

$$
\left|P_{F}\left(K_{q}\right)\right|^{1 / k} \leq c_{1} \max \{\sqrt{q / k}, 1\}\left|B_{2}^{k}\right|^{1 / k},
$$

where $c_{1}>0$ is an absolute constant. In particular,

$$
\left|K_{q}\right|^{1 / n} \leq c_{1}\left|B_{2}^{n}\right|^{1 / n} .
$$

The last ingredient of the proof is a consequence of the main result in 16]: from (1.7) it follows that

$$
\left(\int_{K}\|y\|_{2}^{q} d y\right)^{1 / q} \leq c \sqrt{n} L_{K}
$$

for all $1 \leq q \leq \sqrt{n}$. Since

$$
w\left(Z_{q}(K)\right) \leq\left(\int_{S^{n-1}} \int_{K}|\langle y, \theta\rangle|^{q} d y \sigma(d \theta)\right)^{1 / q} \leq \frac{C \sqrt{q}}{\sqrt{n}}\left(\int_{K}\|y\|_{2}^{q} d y\right)^{1 / q}
$$

for all $1 \leq q \leq n$, we have the following lemma.

Lemma 2.5. Let $K$ be an isotropic convex body in $\mathbb{R}^{n}$. If $1 \leq q \leq \sqrt{n}$, then

$$
w\left(K_{q}\right) \leq C,
$$

where $C>0$ is an absolute constant.

Remark 2.6. Without using Lemma 2.5, which fully exploits the results of [16, we can prove Theorem 1.1 with $\tau=2+\epsilon$ for any $\epsilon>0$.

\section{Covering numbers of $K_{q}$}

Let $N\left(K_{q}, s B_{2}^{n}\right)$ denote the minimal number of translates of $s B_{2}^{n}$ whose union covers $K_{q}$. A standard way to estimate the covering number $N\left(K_{q}, s B_{2}^{n}\right)$ is through the inequality

$$
\left|t B_{2}^{n}\right| \cdot N\left(K_{q}, 2 t B_{2}^{n}\right) \leq\left|K_{q}+t B_{2}^{n}\right|,
$$

which is valid for every $t>0$. We will use our information on the projections of $K_{q}$ in order to give an upper bound for $\left|K_{q}+t B_{2}^{n}\right|$.

Proposition 3.1. Let $K$ be an isotropic convex body in $\mathbb{R}^{n}$. For every $1 \leq q \leq n$ and every $t>0$, we have that

$$
N\left(K_{q}, 2 t B_{2}^{n}\right) \leq 2 \exp \left(C \frac{\sqrt{q n}}{\sqrt{t}}+C \frac{n}{t}\right),
$$

where $C>0$ is an absolute constant. 
Proof. From the classical Steiner formula we know that

$$
\left|K_{q}+t B_{2}^{n}\right|=\sum_{k=0}^{n}\left(\begin{array}{l}
n \\
k
\end{array}\right) W_{[n-k]}\left(K_{q}\right) t^{n-k}
$$

for all $t>0$, where $W_{[n-k]}\left(K_{q}\right)$ is the mixed volume $V_{k}\left(K_{q}\right)=V\left(K_{q} ; k, B_{2}^{n} ; n-k\right)$ (see [18]).

We will use Kubota's integral formula to express $W_{[n-k]}\left(K_{q}\right)$ as an average of the volumes of the $k$-dimensional projections of $K_{q}$ : for every $1 \leq k \leq n-1$ we have

$$
W_{[n-k]}\left(K_{q}\right)=\frac{\left|B_{2}^{n}\right|}{\left|B_{2}^{k}\right|} \int_{G_{n, k}}\left|P_{F}\left(K_{q}\right)\right| d \mu_{n, k}(F) .
$$

Using (3.3), (3.4) and the estimates from Theorem 2.4, we can write

$$
\left|K_{q}+t B_{2}^{n}\right| \leq\left|B_{2}^{n}\right| \sum_{k=0}^{n}\left(\begin{array}{l}
n \\
k
\end{array}\right)\left(c_{1} \max \{\sqrt{q / k}, 1\}\right)^{k} t^{n-k} .
$$

Then, (3.1) shows that

$$
N\left(K_{q}, 2 t B_{2}^{n}\right) \leq \sum_{k=0}^{q}\left(\frac{c_{2} n \sqrt{q}}{k^{3 / 2} t}\right)^{k}+\sum_{k=q+1}^{n}\left(\frac{c_{2} n}{k t}\right)^{k} .
$$

Observe that for $1 \leq k \leq q$ we have

$$
\left(\frac{c_{2} n \sqrt{q}}{k^{3 / 2} t}\right)^{k} \leq\left(\frac{c_{2} n q}{k^{2} t}\right)^{k} \leq \frac{\left(c_{3} \sqrt{n q / t}\right)^{2 k}}{(2 k) !},
$$

while, for $q \leq k \leq n$ we have

$$
\left(\frac{c_{2} n}{k t}\right)^{k} \leq \frac{\left(c_{4} n / t\right)^{k}}{k !}
$$

It follows that

$$
N\left(K_{q}, 2 t B_{2}^{n}\right) \leq \exp \left(c_{3} \frac{\sqrt{q n}}{\sqrt{t}}\right)+\exp \left(c_{4} \frac{n}{t}\right),
$$

and the result follows by the elementary inequality $x+y \leq 2 x y$ for $x, y \geq 1$.

Remark 3.2. The proof actually gives $N\left(K_{q}, 2 t B_{2}^{n}\right) \leq \exp \left(C \frac{n^{2 / 3} q^{1 / 3}}{t^{2 / 3}}+C \frac{n}{t}\right)$ for every $t>0$, but this would play no role in the proof of the main result.

\section{Proof of the theorem}

Let $K$ be an isotropic convex body in $\mathbb{R}^{n}$. Consider the convex body

$$
T=\operatorname{conv}\left(\bigcup_{i=1}^{\left\lfloor\log _{2} n\right\rfloor} \frac{1}{i} K_{2^{i}}\right) .
$$

We will use the following standard fact.

Lemma 4.1. Let $A_{1}, \ldots, A_{s}$ be subsets of $R B_{2}^{n}$. For every $t>0$ we have that

$$
N\left(\operatorname{conv}\left(A_{1} \cup \cdots \cup A_{s}\right), 2 t B_{2}^{n}\right) \leq\left(\frac{c R}{t}\right)^{s} \prod_{i=1}^{s} N\left(A_{i}, t B_{2}^{n}\right) .
$$


Sketch of the proof. For $i=1, \ldots, s$, let $N_{i}$ be a subset of $\mathbb{R}^{n}$ with cardinality $\left|N_{i}\right|=N\left(A_{i}, t B_{2}^{n}\right)$, so that $A_{i} \subseteq \bigcup_{x_{i} \in N_{i}}\left(x_{i}+t B_{2}^{n}\right)$. Let $B_{1}^{s}$ denote the unit ball of $\ell_{1}^{s}$ and fix $Z \subseteq B_{1}^{s}$ of minimal cardinality, so that $B_{1}^{s} \subseteq \bigcup_{z \in Z}\left(z+(t / R) B_{1}^{n}\right)$. It is well-known that $|Z| \leq(c R / t)^{s}$, where $c>0$ is an absolute constant. Consider the set $N=\left\{w=z_{1} x_{1}+\cdots+z_{s} x_{s}: x_{i} \in N_{i}, z=\left(z_{1}, \ldots, z_{s}\right) \in Z\right\}$. Then, $\operatorname{conv}\left(A_{1} \cup \cdots \cup A_{s}\right) \subseteq \bigcup_{w \in N}\left(w+2 t B_{2}^{n}\right)$.

Let $s=\left\lfloor\log _{2} n\right\rfloor$ and $m=\left\lfloor\log _{2}(\sqrt{n})\right\rfloor \simeq s / 2$. We apply Lemma 4.1 with $A_{i}=$ $\frac{1}{i} K_{2^{i}}, 1 \leq i \leq s$, and $t=1$. Observe that $A_{i} \subseteq c_{1} \sqrt{n} B_{2}^{n}$ for all $i \leq s$ (to see this, recall the known fact that if $K$ is an isotropic convex body in $\mathbb{R}^{n}$, then $\left.K \subseteq\left(c n L_{K}\right) B_{2}^{n}\right)$. Using Sudakov's inequality (see [17]) and Lemma 2.5 we see that, since $2^{i} \leq \sqrt{n}$ for $i \leq m$, we have

$$
N\left(A_{i}, B_{2}^{n}\right)=N\left(K_{2^{i}}, i B_{2}^{n}\right) \leq \exp \left(c^{\prime} n w^{2}\left(K_{2^{i}}\right) / i^{2}\right) \leq \exp \left(c_{1} n / i^{2}\right)
$$

for all $i=1, \ldots, m$. Using also the entropy estimates of Section 3 to estimate $N\left(A_{i}, B_{2}^{n}\right)$ for $m<i \leq s=\left\lfloor\log _{2} n\right\rfloor$, we may write

$$
\begin{aligned}
N\left(T, B_{2}^{n}\right) & \leq\left(c_{2} \sqrt{n}\right)^{\left\lfloor\log _{2} n\right\rfloor}\left[\prod_{i=1}^{\left\lfloor\log _{2} n\right\rfloor} N\left(K_{2^{i}}, i B_{2}^{n}\right)\right] \\
& \leq e^{c_{3} n} \exp \left(C \sqrt{n} \sum_{i=m+1}^{\left\lfloor\log _{2} n\right\rfloor} 2^{i / 2}\right) \times \exp \left(C n \cdot\left(\sum_{i=1}^{m} \frac{1}{i^{2}}+\sum_{i=m+1}^{2 m} \frac{1}{i}\right)\right) \\
& \leq e^{c n} .
\end{aligned}
$$

It follows that $|T| \leq\left|C B_{2}^{n}\right|$, where $C>0$ is an absolute constant. Therefore, there exists $x \neq 0$ such that

$$
h_{T}(x) \leq C\|x\|_{2},
$$

and hence,

$$
\|\langle\cdot, x\rangle\|_{2^{i}} \leq C 2^{i / 2} i L_{K}\|x\|_{2}
$$

for every $i=1,2, \ldots,\left\lfloor\log _{2} n\right\rfloor$. This easily implies the following.

Theorem 4.2. Let $K$ be an isotropic convex body in $\mathbb{R}^{n}$. There exists $\theta \in S^{n-1}$ such that

$$
\|\langle\cdot, \theta\rangle\|_{q} \leq C \sqrt{q} \log q\|\langle\cdot, \theta\rangle\|_{2}
$$

for every $q \geq 2$, where $C>0$ is an absolute constant.

A standard argument shows that Theorem 4.2 implies Theorem 1.1 (it is actually equivalent to Theorem 1.1 with $\tau=2$ ).

Remark 4.3. The proof of Theorem 4.2 carries over to the case of an arbitrary log-concave measure: the approach of [16] and all the arguments we have used in this note depend only on the Brunn-Minkowski theory. It follows that if $\mu$ is an isotropic $\log$-concave measure in $\mathbb{R}^{n}$, then there exists $\theta \in S^{n-1}$ such that

$$
\|\langle\cdot, \theta\rangle\|_{L^{q}(\mu)} \leq C \sqrt{q} \log q\|\langle\cdot, \theta\rangle\|_{L^{2}(\mu)}
$$

for all $2 \leq q \leq n$, where $C>0$ is an absolute constant. 


\section{REFERENCES}

1. K. M. Ball, Logarithmically concave functions and sections of convex sets in $\mathbb{R}^{n}$, Studia Math. 88 (1988), 69-84. MR0932007 (89e:52002)

2. S. G. Bobkov and F. L. Nazarov, On convex bodies and log-concave probability measures with unconditional basis, Geom. Aspects of Funct. Analysis (Milman-Schechtman eds.), Lecture Notes in Math. 1807 (2003), 53-69. MR2083388 (2005k:60058)

3. S. G. Bobkov and F. L. Nazarov, Large deviations of typical linear functionals on a convex body with unconditional basis, Stochastic Inequalities and Applications, Progr. Probab. 56, Birkhauser, Basel, 2003, 3-13. MR2073422 (2005f:52013)

4. J. Bourgain, On the distribution of polynomials on high dimensional convex sets, Geom. Aspects of Funct. Analysis (Lindenstrauss-Milman eds.), Lecture Notes in Math. 1469 (1991), 127-137. MR:1122617 (92j:52007)

5. J. Bourgain, On the isotropy constant for $\psi_{2}$-bodies, Geom. Aspects of Funct. Analysis (Milman-Schechtman eds.), Lecture Notes in Math. 1807 (2003), 114-121. MR2083391 (2006c:46011)

6. J. Bourgain, B. Klartag and V. D. Milman, Symmetrization and isotropic constants of convex bodies, Geom. Aspects of Funct. Analysis (Milman-Schechtman eds.), Lecture Notes in Math. 1850 (2004), 101-115. MR2087154 (2005i:52005)

7. S. Campi and P. Gronchi, The $L^{p}$-Busemann-Petty centroid inequality, Adv. in Math. 167 (2002), 128-141. MR.1901248(2003e:52011)

8. B. Klartag, On convex perturbations with a bounded isotropic constant, Geom. Funct. Anal. (2006), to appear.

9. B. Klartag, Uniform almost sub-gaussian estimates for linear functionals on convex sets, Preprint.

10. E. Lutwak and G. Zhang, Blaschke-Santaló inequalities, J. Differential Geom. 47 (1997), 1-16. MR.1601426 (2000c:52011)

11. E. Lutwak, D. Yang and G. Zhang, $L^{p}$ affine isoperimetric inequalities, J. Differential Geom. 56 (2000), 111-132. MR1863023 (2002h:52011)

12. V.D. Milman and A. Pajor, Isotropic position and inertia ellipsoids and zonoids of the unit ball of a normed n-dimensional space, Geom. Aspects of Funct. Analysis (LindenstraussMilman eds.), Lecture Notes in Math. 1376 (1989), 64-104. MR1008717 (90g:52003)

13. V.D. Milman and G. Schechtman, Asymptotic Theory of Finite Dimensional Normed Spaces, Lecture Notes in Math. 1200 (1986), Springer, Berlin. MR0856576 (87m:46038)

14. G. Paouris, $\Psi_{2}$-estimates for linear functionals on zonoids, Geom. Aspects of Funct. Analysis (Milman-Schechtman eds.), Lecture Notes in Math. 1807 (2003), 211-222. MR2083399 (2005g:52021)

15. G. Paouris, On the $\Psi_{2}$-behavior of linear functionals on isotropic convex bodies, Studia Math. 168 (2005), no. 3, 285-299. MR2146128(2006c:52004)

16. G. Paouris, Concentration of mass on convex bodies, Geom. Funct. Anal. (2006), to appear.

17. G. Pisier, The Volume of Convex Bodies and Banach Space Geometry, Cambridge Tracts in Mathematics 94 (1989). MR1036275 (91d:52005)

18. R. Schneider, Convex Bodies: The Brunn-Minkowski Theory, Encyclopedia of Mathematics and its Applications 44, Cambridge University Press, Cambridge (1993). MR1216521 (94d:52007)

Department of Mathematics, University of Athens, Panepistimiopolis 157 84, Athens, Greece

E-mail address: apgiannop@math.uoa.gr

Équipe d’Analyse et de Mathématiques Appliquées, Université de Marne-La-Vallée, Champs sur Marne, 77454, Marne-la-Vallée, Cedex 2, France

E-mail address: Alain.Pajor@univ-mlv.fr

Équipe d'Analyse et de Mathématiques Appliquées, Université de Marne-La-Vallée, Champs sur Marne, 77454, Marne-La-Vallée, Cedex 2, France

E-mail address: grigoris_paouris@yahoo.co.uk 